\title{
Miura-ori enabled stretchable circuit boards
}

\author{
Yongkai Li $\mathbb{D}^{1,2}$, Weixuan Liu $\mathbb{D}^{3}$, Yang Deng ${ }^{1}$, Wei Hong $\mathbb{D}^{3 凶}$ and Hongyu Yu $\mathbb{D}^{1 凶}$
}

Origami, an ancient form of papercraft, provides a way to develop functional structures for engineering applications. In this paper, we report an approach to design and manufacture a stretchable circuit board (SCB) with origami structures. The benefits of developable, flat-foldable, and rigid-foldable origami-based structures as SCBs are discussed, and a representative structure, Miura fold (or Miura-ori), is chosen to be investigated. Under the constraints induced by the mounted components' dimensions, the Miura-ori structures for specific applications can be defined. We propose three methods for better fabrication, including direct folding, stiffness modification, and kirigami enhancement, to improve a planar sheet's foldability. A wearable ECG (electrocardiogram) system based on MO-SCB (Miura-ori enabled SCB) technology is built, and the stretchable portion is made of commercial FPCBs (flexible printed circuit board), providing desired stretchability and reliability. The proposed technology routine is compatible with industrial production and may pave the application of stretchable electronics.

npj Flexible Electronics (2021)5:3 ; https://doi.org/10.1038/s41528-021-00099-8

\section{INTRODUCTION}

As the critical component of a stretchable sensing system, stretchable electronic circuits have emerged as an attractive technology over the past decades due to the high potential in industrial applications, such as wearable electronics ${ }^{1-3}$ and medical electronics ${ }^{1,3,4}$. Since the early 2000 s, various materials, such as elastic polymer substrates ${ }^{5-7}$, flexible composite ${ }^{8,9}$, and liquid conductors ${ }^{10-12}$, have been explored. Wagner et al. ${ }^{13}$ and Rogers et al. ${ }^{14,15}$ proposed a method combining rigid arrays and wavy or serpentine interconnections to form stretchable circuits, by which they could reduce the maximum stress and effectively extend the lifetime of the products. Nevertheless, the wavy or serpentine interconnections demand complicated manufacturing processes and, in most cases, are not compatible with standard industrial production. Moreover, the sizeable space occupied by the two-dimensional interconnections lacks functionality, wastes the active area, and increases the cost, restricting further commercialization ${ }^{12,16}$.

Origami-based approaches potentially outperform the limits of the above methods. Origami is an ancient form of papercraft in which, following predesigned patterns, two-dimensional sheets are folded into compact and deformable three-dimensional structures $^{17}$. A widely recognized origami form, Miura fold (or Miura-ori), which has various desired properties, including stretchability and rigid foldability, has been used in engineering, architecture, and artistic design ${ }^{17-20}$. The strain is effectively suppressed in the rigid facet areas with the deformation mainly localized on the creases, making the Miura-ori compatible with rigid or nonflexible components ${ }^{21,22}$. To our knowledge, very few studies $^{21}$ have explored the potential application of the origami structure in stretchable displays, and no systematic research has been carried out on complex SCBs with multiple components installed for system-level sensing applications.

In this study, we utilize the Miura-ori for a stretchable sensing system by placing rigid components in the facets and identify the geometric constraints of a Miura-ori configuration for specific circuits with various components under the premise of a certain size and certain stretchability. Three methods enhancing the foldability of the creases during the manufacture of the MO-SCB are proposed to realize the above configuration and decrease the strain concentration. An integrated ECG monitoring system is built using a MO-SCB, demonstrating that the MO-SCB is suitable for carrying complex circuits and rigid components.

\section{RESULTS AND DISCUSSION} Compatibility between origami structures and circuit boards

Developability, flat-foldability, and rigid foldability are crucial characteristics of an origami pattern ${ }^{23}$, revealing compatibility with the origami-based circuit boards. Nomenclatures and symbols related to the origami structures are defined in Supplementary Note $1^{24,25}$.

- Developability is the condition of having an initial state with zero Gaussian curvature at every points ${ }^{26}$. The feature of developable origami is that it could be constructed by bending a planar surface according to the predesigned pattern without requiring extensional deformation ${ }^{27}$. It is necessary because the origami-based circuit board is made from a planar sheet, such as an FPCB.

- Flat-foldability is the condition of having a flat-, fully folded state $^{26}$. Locally, by satisfying Maekawa's theorem ${ }^{28}$ and Kawasaki's theorem ${ }^{29}$, a flat-foldable degree-4-vertex (D4V), which are fundamental for Miura-ori patterns, can be flattened without being crumpled, bent, or damaged; globally, a flatfoldable origami pattern avoids self-collision of the sheet during the folding procedure by employing locally flatfoldable D4Vs everywhere ${ }^{30}$. Although globally flat-foldability is not a must due to the mounted components' finite thickness, it is preferred for a more compact structure and higher deformability.

- Rigid foldability is the condition implying that no deformation in facets and crease lines is induced during folding/unfolding $^{26}$. Ideally, rigid-foldable origami can realize a development mechanism even if the facets are all rigid panels ${ }^{31}$. It is a vital

\footnotetext{
${ }^{1}$ Department of Mechanical and Aerospace Engineering, The Hong Kong University of Science and Technology, Clear Water Bay, Hong Kong, China. ${ }^{2}$ Smart Manufacturing Thrust, Systems Hub, The Hong Kong University of Science and Technology (Guangzhou), Nansha, Guangzhou, Guangdong, China. ${ }^{3}$ Department of Mechanics and Aerospace Engineering, Southern University of Science and Technology, Shenzhen, Guangdong, China. ${ }^{凶}$ email: hongw@sustech.edu.cn; hongyuyu@ust.hk
} 
feature for the origami-based circuit board because it exerts minimal strain to the mounted components during deformation and extends the device's lifespan.

\section{Geometry analysis of MO-SCBs}

As a representative structure of origami, Miura-ori is employed to design the MO-SCB. The ridge lengths $a$ and $b$, and the folding degree $\varphi(0 \leq \varphi \leq 2 \beta$, defining the reduction of the zig angle or zag angle of the major creases during folding) can parameterize a cell of the Miura-ori structure, as shown in Fig. 1a. A full Miura-ori (Fig. 1b) consists of an array of such unit cells, and its overall dimensions: length $L$, width $W$, and height $H$, can be expressed with the above four parameters and the number of facets $\mathrm{N}_{l} \times \mathrm{N}_{w}$ (detailed in Supplementary Note 1).

The linear stretchability of a MO-SCB is defined as $\varepsilon^{\prime}=\frac{L_{\max }-L_{\min }}{L_{\min }}$ and $\varepsilon^{W}=\frac{W_{\max }-W_{\min }}{W_{\min }}$, along with the major and minor crease directions (Fig. $1 c$ ), respectively, where symbols with subscripts max and min are the corresponding maximum and minimum dimensions. Some engineering considerations like residual deformation $^{32,33}$, sheet thickness ${ }^{34-36}$, and external forces ${ }^{36}$ reduce the range of the folding degree $\varphi$ in a Miura-ori design (Fig. 2c).

- Ideal folding range (RANGE I): For an ideal Miura-ori structure with negligible sheet thickness and bending radius, the maximum and minimum in-plane dimensions are taken to be those in the flat-folded state ${ }^{36}$ and fully unfolded state, respectively.
- Unpackaged bare MO-SCB folding range (RANGE II): However, inelastic materials, such as plastics and metals, have small residual deformation and cannot return to the original flat state (Fig. 2a-l) after folding, even when fully relaxed. An additional parameter defining the relaxed state of a practical $\mathrm{MO}-\mathrm{SCB}$ - the residual folding degree $\varphi_{0}$ is introduced to characterize the residual deformation and is used to evaluate $L_{\max }$ and $W_{\max }$ (Fig. 2a-III). Moreover, due to the finite sheet thickness and elastic deformation, the ultimate folding state is $\varphi=\varphi_{\mathrm{c}}$ (most compact state, Fig. 2a-IV) instead of the ideal flatfolded state $\varphi=2 \beta$. Consequently, the folding status of a bare $\mathrm{MO}-\mathrm{SCB}$ ranges from a residual deformation state to the most compact state.

- Elastomer encapsulated MO-SCB folding range (RANGE III): Furthermore, an incompressible elastomer encapsulates the Miura-ori structure (Fig. 2b), thereby protecting the MO-SCB and the electronic components but limiting the practical minimum in-plane dimensions $L_{\min }$ and $W_{\min }$. The Miura-ori structure is molded to the stable folding state $\left(\varphi=\varphi_{m}\right.$, Fig. 2a-II), with a specific dimension, an acceptable stretchability, and sufficient space to install electronic components. Thus, the folding range of a packaged MO-SCB shrinks-from a residual deformation state to a stable folding state, compared with a bare MO-SCB.

Unlike other Miura-ori enabled stretchable structures ${ }^{17,19,21}$, MOSCBs carry electronic components on the facets' surface, leading to a geometric constraint due to the components' finite thickness and
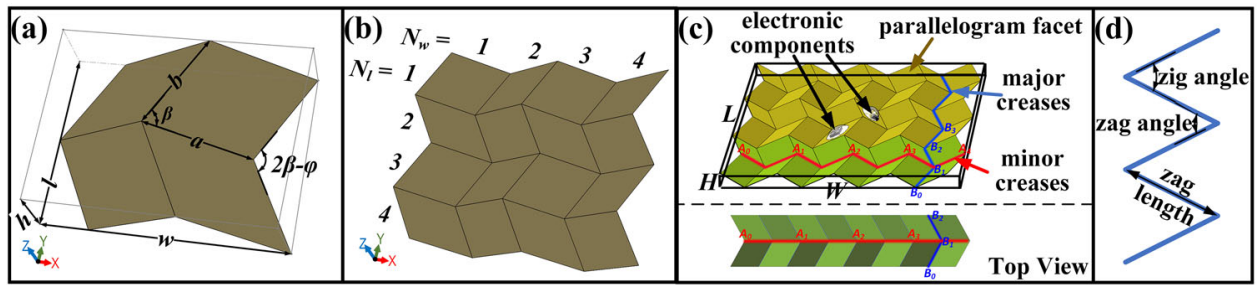

Fig. 1 Geometry of the Miura-ori structure. Schematics of $\mathbf{a}$ a unit cell of the Miura-ori, $\mathbf{b}$ a full Miura-ori structure, $\mathbf{c}$ a MO-SCB, and $\mathbf{d}$ the major creases of the pattern in zigzag. In $\mathbf{b}, N_{l}=4$ and $N_{w}=4$; in $\mathbf{c}, N_{l}=6$ and $N_{w}=8$. The minor(red) and major(blue) creases are defined based on parities, detailed in Supplementary Note 1. The top view of part of the structure (green area) in c shows that the projection of the minor crease (red) is collinear. For the major creases, the zig angle equals the zag angle, and the zag length equals the ridge length $b$.

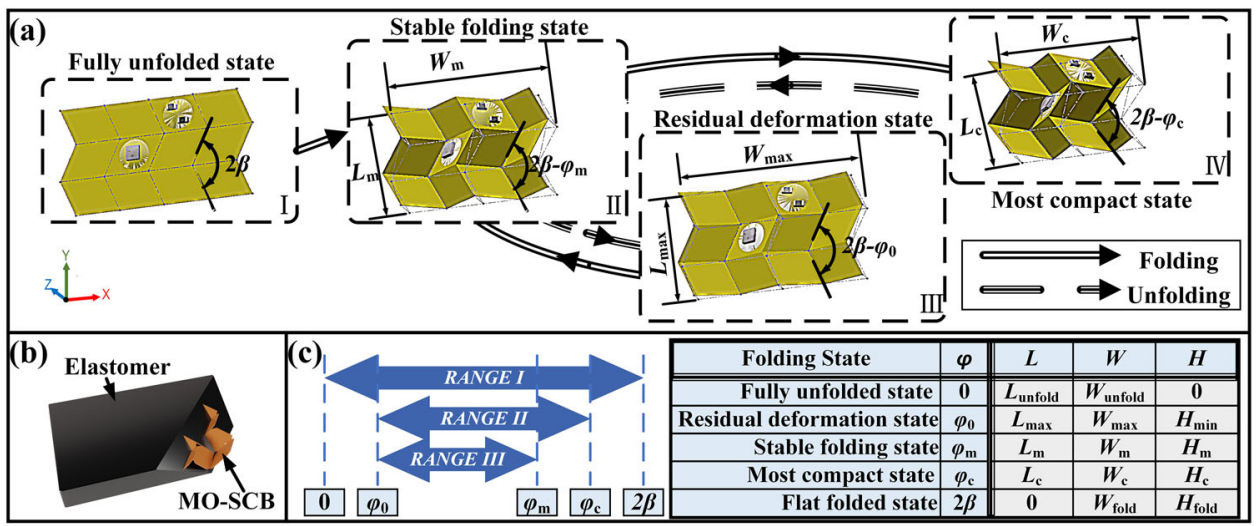

Fig. 2 Deformation of the Miura-ori patterned sheet. a Schematics of the MO-SCB in different folding states (where $N_{l}=3$ and $\left.N_{w}=4\right)$. (I) fully unfolded state, $\varphi=0$ (i.e., before any process); (II) stable folding state, $\varphi=\varphi_{\mathrm{m}}$ (i.e., the state when packaging); (III) residual deformation state, $\varphi=\varphi_{0}$ (i.e., relaxed state with residual deformation); (IV) most compact state, $\varphi=\varphi_{\mathrm{c}}$. b Schematic of a packaged MO-SCB. c Folding degrees $\varphi$ in different folding states. RANGE I: folding ranges from a fully unfolded state to a flat-folded state, requiring negligible thickness and bending radius. RANGE II: folding ranges from a residual deformation state to the most compact state; both the finite thickness and the elastic deformation of the MO-SCB lead to the reduction of the range of the folding degree $\varphi$ comparing with RANGE I. RANGE III: folding ranges from residual deformation state to stable folding state; incompressible packaging material (such as elastomer) protects the MO-SCB while reducing the deformation range comparing with RANGE II. 


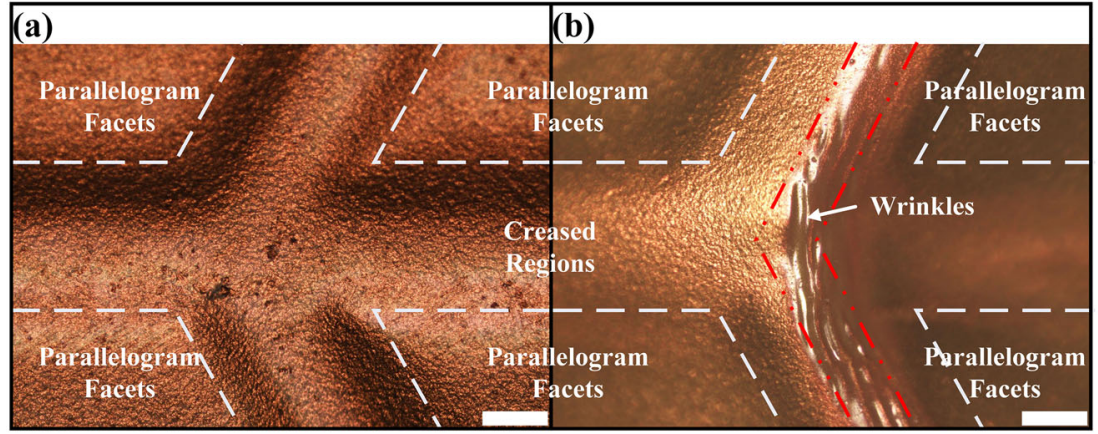

Fig. 3 Wrinkles occur in the crease area. $\mathbf{a}$ Before and $\mathbf{b}$ after direct folding. Scale bar: $300 \mu \mathrm{m}$.

dimensions. The optimized design has been conducted to avoid the collision (or self-intersection in geometry) either between a component and MO-SCB surface (Supplementary Fig. 2b) or between components on neighboring facets (Supplementary Fig. 2c), which is discussed in Supplementary Note 2.

\section{Enhancing the foldability of the MO-SCB}

After several undesired designs (detailed in Supplementary Note 3 ), we realized the importance of developing a process to enhance substrate foldability. Unlike folding an origami structure whose creases have zero bending stiffness, it is almost impossible to fold a uniform plate without pretreated creases according to a designed pattern. Thus, three methods, including direct folding, stiffness modification, and kirigami enhancement, are proposed to improve a planar sheet's foldability to produce MO-SCB.

The most straightforward method is to apply concentrated forces at the creases, e.g., by pressing with a set of rigid molds, as crease pretreating. The advantages of this method include low fabrication complexity and no additional processing. Wrinkles which occur in the crease areas (Fig. 3), together with plastic deformation, facilitate the subsequent folding ${ }^{37,38}$. However, it is quite hard to control the range of the wrinkles precisely, especially when the sheet is thick and/or the folding degree $\varphi$ is large. Therefore, the method alone is not sufficient and employed to provide the initial direction, i.e., the folds' parity.

Stiffness modification, either stiffening the parallelogram facets or weakening the crease areas' bending stiffness, can improve the foldability. Noticing that the bending stiffness $K$ scales with Young's modulus $E$, the width of the plate $b$ (not the parameter defining Miura-ori structure), and the cubed of the thickness $t$, i.e., $K \propto E b t^{3}$, we can modify the rigidity of the crease areas by reducing the thickness (reducing $t$ ), changing the material to a flexible one (reducing $E$ ), and removing parts of the region (reducing $b$ ).

Numerical simulation with SIMULIA Abaqus (detailed in Supplementary Note 4) based on the finite element method (FEM) is carried out to study bending stiffness modification on the folding process. Initially, the sheet is in a state of small residual deformation $\left(\varphi_{0, \text { sim }}=6^{\circ}\right.$, after the stage- 1 folding $)$. Figure $4 a$ shows snapshots of the representative states of deformation of the structures of two thickness ratios, $t_{\text {crease }} / t_{\text {facet }}=0.05$ and 0.39 , under different levels of lateral compression, while the complete deformation processes can be viewed in Supplementary Movie 1, in which the color scale indicates the strain-energy density. The sheet with a given thickness ratio (e.g., 0.39, Supplementary Movie 1b) experiences two modes of deformation while compressed, the origami-dominated mode (or bent Miura-ori ${ }^{39,40}$ ) and the globalbuckling-dominated one ${ }^{39,41}$. The transition between the two modes may break the Miura-ori structure's symmetry, leading to irreversible damage to the MO-SCB. To study such transition, we investigate the total strain energies (Fig. 4b) and structural deformations of both a constrained Miura fold (Supplementary Movie 2a) and one condition in a global-buckling mode (Supplementary Movie 2b), aside from the free-standing structure shown in Fig. 4a. It is hypothesized that the transition happens when the structure's energy in the buckling-dominated phase is lower than that in the origami-dominated one, and the structure is sufficiently perturbed to overcome any energy barrier ${ }^{42}$. After the critical point in Fig. 4b, the global-buckling structure's energy is lower than that of the constrained Miura fold, and a mode transition may occur under perturbation. We construct the morphological phase diagram in Fig. $4 \mathrm{c}$ for the equivalent thickness ratio ranging from 5 to $40 \%$, and the elastic modulus $E$ held constant. The lower region (low stiffness ratio or low relative compression) corresponds to the origami-dominated mode, while the upper right (higher crease stiffness and more extensive compression) is likely to be dominated by buckling. Although the transition does not necessarily occur due to the existence of a finite energy barrier, we decided to avoid it for safety. The range of the origami-dominated zone is inversely correlated to the bending stiffness ratio. Therefore, the more significant modification in bending stiffness, the more extensive the manageable range in a folding process.

With the planar sheet's bending stiffness, the equivalent thickness ratios near the major and minor creases are derived in Supplementary Note 5, corresponding to the gray dashed lines in Fig. 4c. The specially-designed crease areas provide enough region to ensure that the stable folding degree $\varphi_{\mathrm{m}}\left(21^{\circ}\right.$, i.e., $\Delta W=$ $4.36 \mathrm{~mm}$ ) falls into the origami-dominated mode, which is consistent with the experiment.

Vertexes are the weakest part of the Miura-ori structure due to geometrically sharp angles and the mechanically concentrated strain shown in Fig. 5. With the concept kirigami, which enhances classic origami structures by including cuts (or removal of materials) in addition to folds, we remove some parts not containing electrical connections ${ }^{21}$ near the vertexes, to reduce the stress in the facets where electronic components mount and improve the overall reliability of the system. The distributions of the maximum principal strain (logarithmic, LE) in a traditional Miura-ori structure and in kirigami-enhanced structures with thin slits and holes around the vertexes reveal that the strain at the center of the facets is effectively reduced by over $50 \%$ by employing the method. The strain decrease in the facets reduces the possibility of the debonding between mounted components and substrate. Moreover, the four creases and the four sectors of a vertex do not converge perfectly because of the substrate's finite thickness ${ }^{43}$, which causes the panels' interference. The holes enable the MO-SCB to deform freely ${ }^{21}$ (Supplementary Fig. 8), provide more DOFs (degree-of-freedom), and facilitate the folding process (Supplementary Movie 3).

To our knowledge, although the above principles are used every so often (regarded as employing the direct folding 


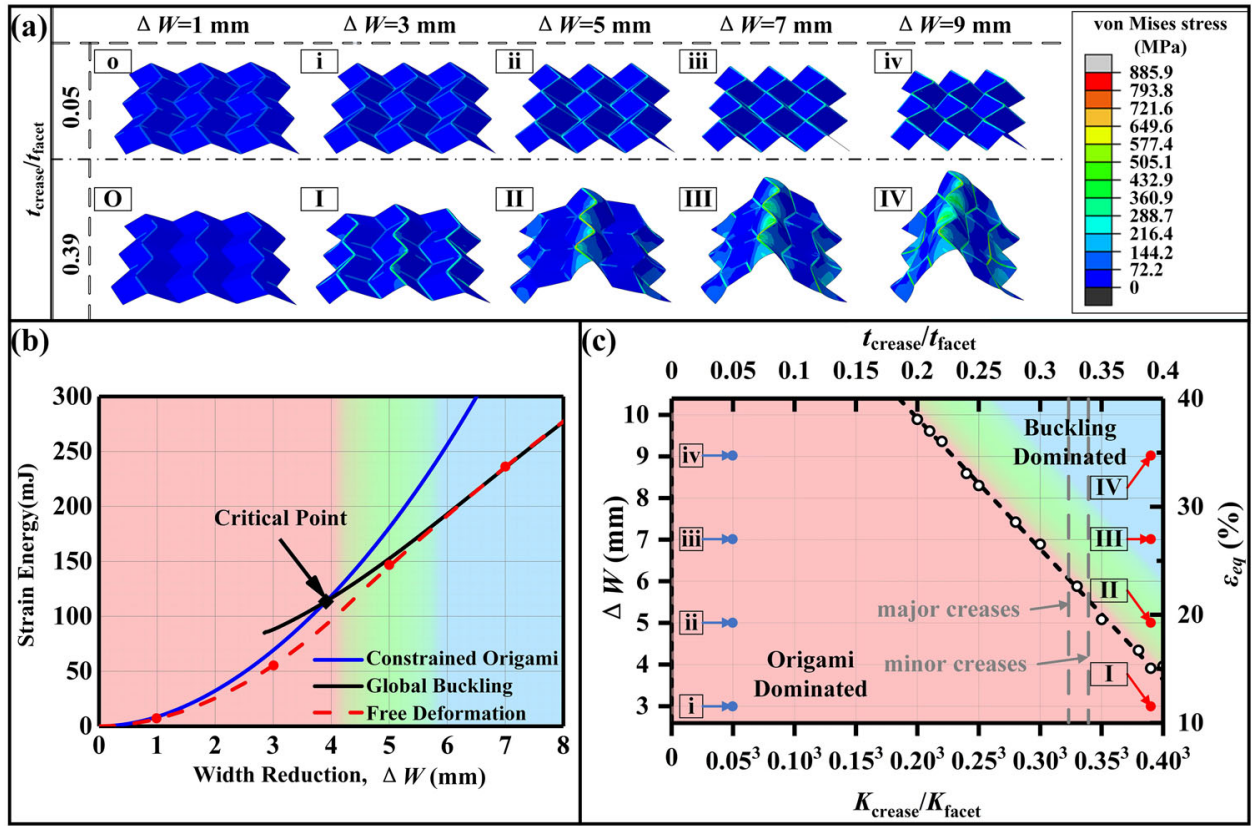

Fig. 4 Simulation for the folding process with different degrees of stiffness modification. a Deformation of a free Miura-ori structure with different thickness ratios when lateral compression (width reduction) $\Delta W=0,1, \ldots, 9 \mathrm{~mm}$, respectively. Deformation with $t_{\text {crease }} / t_{\text {facet }}=0.05$ are in o-iv; Deformation with $t_{\text {crease }} / t_{\text {facet }}=0.39$ are in O-IV. b Strain-energy analysis of the Miura-ori structure with a thickness ratio $t_{\text {crease }} / t_{\text {facet }}$ $=0.39$. The blue and black solid curves refer to the strain energy of a constrained Miura fold and a manually set global-buckling state (curve begins from when $\Delta W=3 \mathrm{~mm}$ ), respectively. These two curves meet at a critical point. The red dashed curve refers to the strain energy of the free Miura-ori deformation in a. c Phase diagram of the folding process with different thickness ratios $t_{\text {crease }} / t_{\text {facet }}$. For the model with the same material (same Young's modulus $E$ ), the bending stiffness $K$ is proportional to the cube of the thickness $t$, i.e., $K \propto t^{3}$. The dashed boundary is the critical points with different bending stiffness ratios $K_{\text {crease }} / K_{\text {facet }}$. Below the boundary, the strain energy of the constrained Miura-ori is lower than that of the global-buckling one, i.e., origami dominated; above the boundary oppositely, i.e., buckling dominated, the structural transition may happen. Overall structure parameters: $a=4.5 \mathrm{~mm}, b=3.9 \mathrm{~mm}, \beta=66^{\circ}, N_{l}=5, N_{w}=6$.

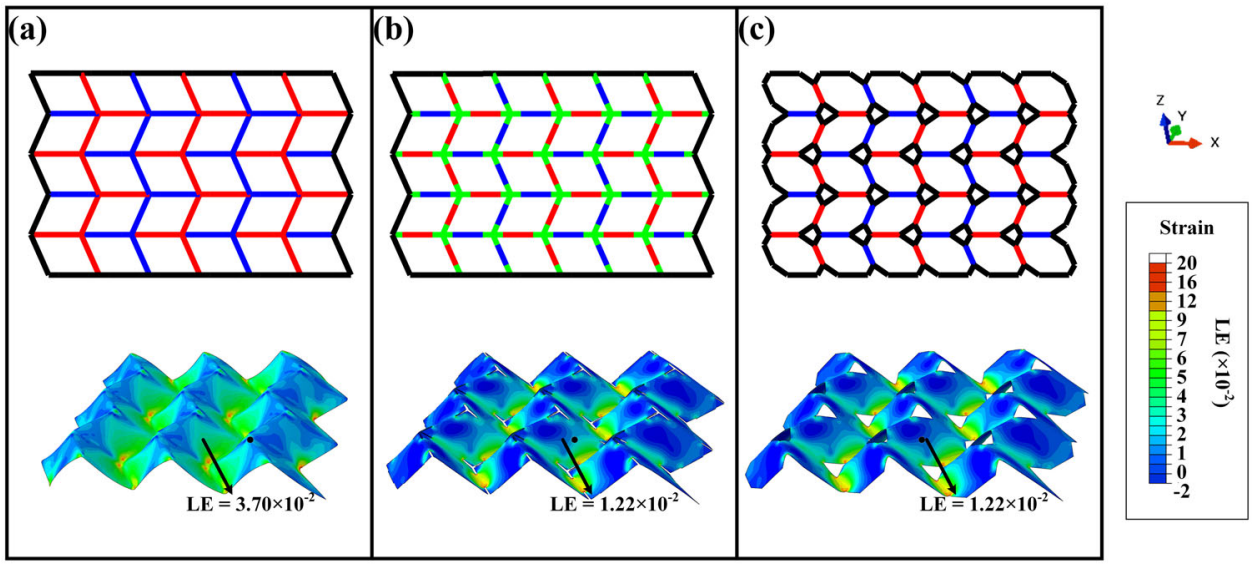

Fig. 5 2D designs and the corresponding distributions of logarithmic strain. The patterns are a the traditional Miura-ori pattern, and $\mathbf{b}$ the kirigami-enhanced pattern with thin slits, and c with kite-shaped holes around the vertexes. Mountain folds are in red; valley folds blue; boundary edges black, and slits green. Structure parameters: $a=4.5 \mathrm{~mm}, b=3.9 \mathrm{~mm}, \beta=66^{\circ}, N_{l}=5, N_{w}=6$, width reduction $\Delta W=3.5 \mathrm{~mm}$.

itself $^{17,40,44-46}$, both direct folding and stiffness modification ${ }^{35,47-50}$, both direct folding and kirigami enhancement ${ }^{51}$, and all the three methods $\left.{ }^{19,21,52,53}\right)$, there is no systematical investigation on them.

The three methods collaborate to enhance the foldability. After employing direct folding, the crease areas' stiffness is reduced due to irreversible deformation such as the wrinkles; the out-of-plane folding (as the stage-1 folding) defines the mountain-valley assignment initially, which is necessary for in-plane pressing (as the stage- 2 folding). The narrower hinges from the kirigami enhancement decrease the equivalent bending stiffness. Besides, the combination of kirigami enhancement and stiffness modification effectively reduces the maximum strain in the facet areas and facilitates the folding process, as discussed in Supplementary Note $6^{54}$.

\section{Mechanical and electrical performances}

After folding, the origami-based portion of the board shrank inplane. The stretchability of the MO-SCB along with minor and major crease directions $\varepsilon^{w}$ and $\varepsilon^{\prime}$ were $33.3 \%$ and $8.7 \%$, 


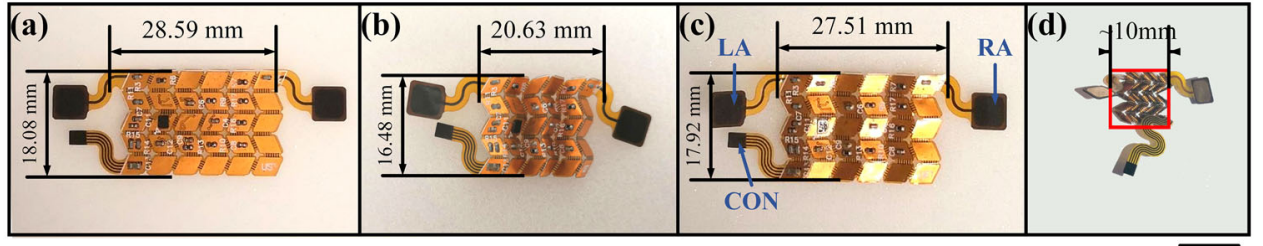

Fig. 6 Dimensions of a bare MO-SCB. A MO-SCB a without folding, $\mathbf{b}$ after the 1 st folding (i.e., the stable folding state), and $\mathbf{c}$ after the $1 \mathrm{st}$ unfolding (i.e., the residual deformation state). d The origami-structured part can be compressed to about $10 \times 10 \mathrm{~mm}^{2}$ square in the plane (i.e., the most compact state). The line CON refers to the connection between the stretchable part and the hard part of the system, including four pins VCC/GND/LOD/OUT. The lines RA (right arm) and LA (left arm) are used for ECG signal collection. Scale bar: $10 \mathrm{~mm}$.

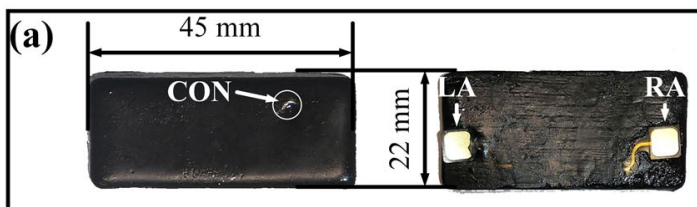

(b)
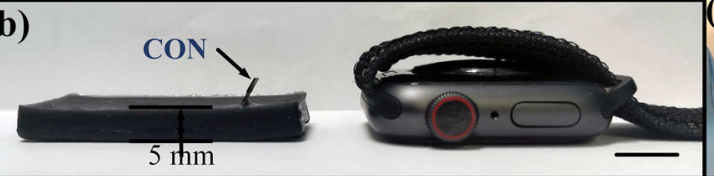

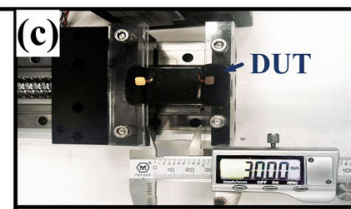

(d)

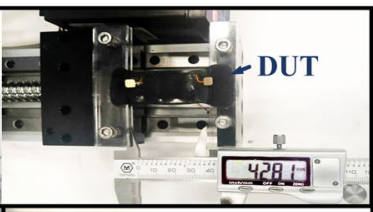

(e)

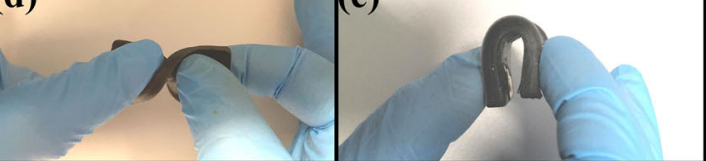

Fig. 7 Mechanical test for the stretchable part of the wearable ECG monitoring system. a The packaged board is viewed from the top and bottom. b The packaged board's thickness compared with an Apple Watch Series 4 (thickness: 10.7 mm). All the dimensions are measured with a micrometer. Scale bar for $\mathbf{a}$ and $\mathbf{b}: 10 \mathrm{~mm}$. c The stretchable portion of the system (device under test, DUT) is tested with a uniaxial tensile testing platform driven by a stepper motor. The actually-tested area is the middle part of the device along the long side, extending from 30.00 to $42.81 \mathrm{~mm}$. The two short edges of the device are both clamped on the platform, so only the device's midpiece is loaded, including the origami-structured portion and its corresponding elastomer package. The packaged MO-SCB can be $\mathbf{d}$ twisted and e bent (Supplementary Movie 4b).

respectively (Fig. 6a-c). Due to more DOFs introduced by the kirigami enhancement and the finite thickness of panels, the actual size did not perfectly match the theoretical one (derived in Supplementary Note 7). The Miura-ori structure enabled the board to be compressed to a $10 \times 10 \mathrm{~mm}^{2}$ square (i.e., over $75 \%$ stretchability) without damaging the circuit (as the most compact state, in Fig. 6d).

The planar sheet was bent in the crease areas, where the amount of strain on particular layers of the structure requires evaluation. Because the flexible materials allow much more elongation than the copper without fracturing ${ }^{55}$, and the strain inside the copper layer is theoretically acceptable, as derived in Supplementary Note 8 , the board should be able to survive in the repeated bending of the crease areas, i.e., repeated deformation of the MO-SCB. An experiment of monitoring the wires in the MOSCBs after finite cycles of deformation was conducted, which verified the wire fatigue or cracks were not generated even after 3000 times cycling (detailed in Supplementary Note 9) ${ }^{21}$.

Both an origami-structured part and three wavy-structured connections of the MO-SCB were packaged. The dimension of the packaged device was $45 \times 22 \times 5 \mathrm{~mm}^{3}$, acceptable for wearable electronics (Fig. 7a-b). The packaged device survived after being stretched by about $40 \%$ along the long side (Fig. 7c), twisted (Fig. 7d), and bent (Fig. 7e). Because the low-modulus package provided extra stretchability besides protection, the device's total stretchability was higher than that of the bare MO-SCB (discussed in Supplementary Note 10). There was no visible degradation (either mechanical or electrical) on the device after 100 cycles of stretching and recovery with a speed of $3 \mathrm{~mm}$ per second and an elongation of $12 \mathrm{~mm}$ (Supplementary Movie 4a).

Figure $8 \mathrm{a}$ shows the frequency response curves of the MO-SCB before folding, after folding, and after packaging, which was consistent with the theoretical value calculated from the official design tool (AD8232/AD8233 Filter Design Tool). Changes in electrical characteristics caused by the board and package's deformation were negligible, as shown in Fig. 8b, where an ECG signal generator (Mingsheng SKX-2000C, $60 \mathrm{bpm}, 1 \mathrm{mV}$ ) was used as the input. The waveform corresponding to the human body test reveals the effectiveness of extracting the heartbeat. The raw data collected was processed by applying a low-pass Butterworth filter (40 Hz cutoff) in MATLAB, and the noise, mainly powerline sourced $(50 \mathrm{~Hz})$, did not affect the extraction of necessary information (such as heart rate) and could be filtered if necessary. Furthermore, the above stretchable part of the system was connected to the rigid part to form portable hardware, and it was paired with a smartphone via BLE (Bluetooth Low Energy, Fig. 8c). The smartphone provided a graphic interface to control the hardware. Figure $8 \mathrm{~d}$ shows the app on which the waveform, average voltage, and sampling position of the ECG signal, the remaining battery, and button control for connection were placed.

\section{MO-SCB: trends and futures}

$\mathrm{MO}-\mathrm{SCBs}$ can realize fast commercialization and pave the application of stretchable electronics. The initial substrate is a commercial FPCB, which is well developed in the industry. Almost all high-performance electronic components are rigid, requiring a low-strain facet's handling (stretchable substrates' shortcoming because of the structural incompatibility ${ }^{5-7}$ ) and a high-density routine (two-dimensional interconnections' disadvantage ${ }^{12,16}$ ), and the proposed MO-SCB is promising to meet the requirements, as discussed in Supplementary Note 11.

Furthermore, the proposed MO-SCB technology can be used in other structures inspired by Miura-ori ${ }^{56-58}$, serving as a Miura-ori family, as described in Supplementary Note 12. It is believed that 


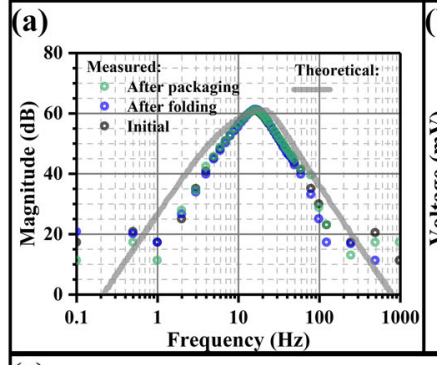

(c)

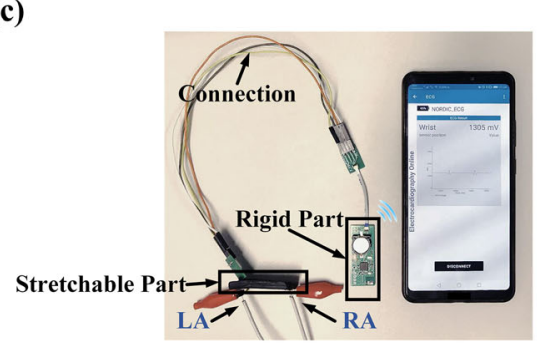

(b)
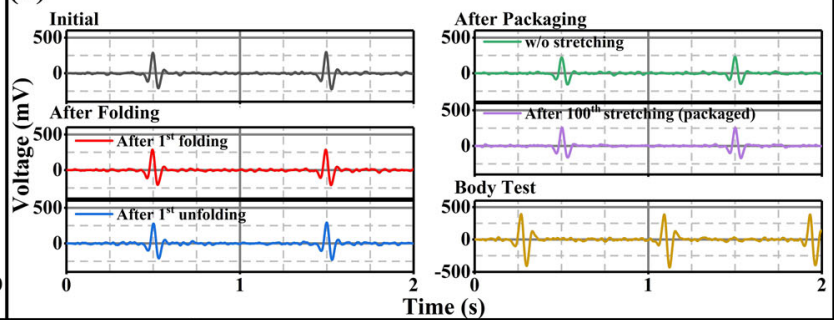

(d)

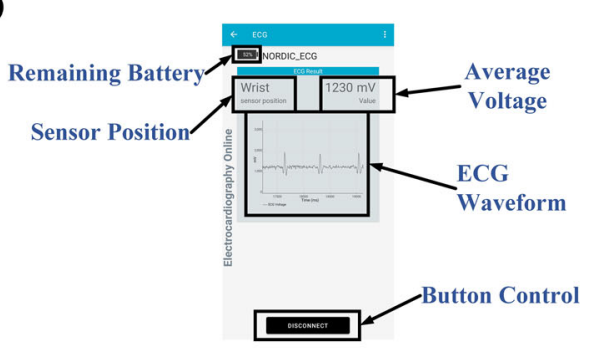

Fig. 8 Electrical test for the MO-SCB. a The frequency response of the MO-SCB (@V $\left.V_{\mathrm{p}-\mathrm{p}, \mathrm{in}}=1 \mathrm{mV}\right)$. The theoretical frequency response is calculated from the Tool provided by Analog Devices (NASDAQ: ADI). b ECG waveforms. Measured with Tektronix-TBS1052B. An ECG signal generator was used as the input of the ECG monitoring module for the waveforms corresponding to initial, after folding, and after packaging. The raw data collected was processed by applying a low-pass Butterworth filter ( $40 \mathrm{~Hz}$ cutoff) in MATLAB. c Schematic of the ECG monitoring system. The ECG signal generator serves as the source. d A screenshot of the Android app showing the outputs. The signal was collected from one of the authors, Mr. LI Yongkai's wrists.

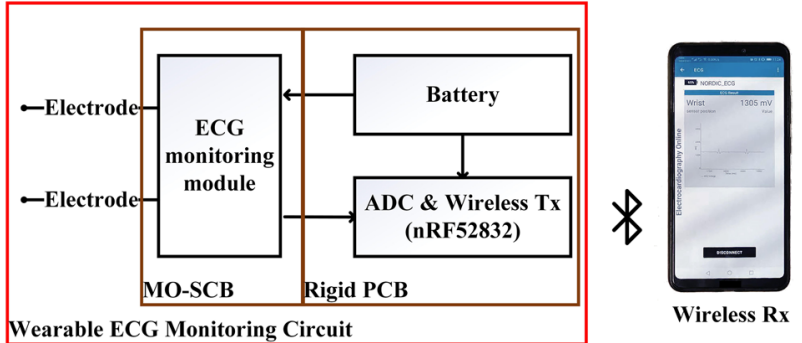

Fig. 9 Block diagram of the ECG monitoring system.

advanced package technologies such as WLCSP (wafer-level chipscale packaging) are leading to smaller-size ICs; the thinner, softer, and more robust FPCBs provide smaller bending radius and better reliability at the creases; and that the origami-based LIBs (Li-ion battery) or supercapacitors are practicable ${ }^{17,19}$. All of them can facilitate the realization of fully origami-based stretchable systems.

\section{METHODS}

\section{System architecture and geometric design}

A wearable ECG monitoring circuit was designed ${ }^{59}$ and manufactured based on the proposed MO-SCB technology, as shown in Fig. 9. It has been revealed that the larger the component size, the larger each parallelogram facet in the Miura-ori pattern, leading to an increase in the Miura-ori structure's height, i.e., the thickness of the MO-SCB. When the SCB is too thick, it may not be acceptable for wearable devices and applications. To balance the thickness of the MO-SCB and the functionality of the system, we only placed the ECG monitoring module on the MO-SCB; other large components, such as the ADC \& Wireless Tx Module, and battery, required a rigid $\mathrm{PCB}$ for their placements. A smartphone could receive and process the collected data and demonstrate the ECG waveforms on the screen.

The Miura-ori pattern was determined according to the size of the most significant component (ADI, AD8233ACBZ) mounted on the MO-SCB. Margins for $x$ and $y$ directions were set to $50 \%$ of the devices' dimensions to ensure robustness. For the MO-SCB in the ECG monitoring system, the constant $N_{l}$ and $N_{w}$ were set to 5 and 6 , respectively. Moreover, the residual folding degree $\varphi_{0}$ was set to $2^{\circ}$ to characterize the residual deformation. To facilitate the circuit board design with computer-aided design software, we chose $[a, b, \beta]=\left[4.5 \mathrm{~mm}, 3.9 \mathrm{~mm}, 66^{\circ}\right]$ as the variables for the pattern; when $\varphi_{\mathrm{m}}=21^{\circ}$, the desired height, and stretchability along the minor crease direction are $3.13 \mathrm{~mm}$ and $28.0 \%$, respectively, as derived in Supplementary Note 7.

\section{Process for fabricating the MO-SCB}

We chose a commercial FPCB as the primary substrate of the MO-SCB. The FPCB consisted of a double-side RA FCCL (rolled-annealed flexible copper clad laminate, thicknesses of polyimide layer and each copper layer was 12 and $18 \mu \mathrm{m}$, respectively), two layers of coverlays (CVLs, SKCKOLONPI GF, $37 \mu \mathrm{m}$ including adhesive), and several stiffeners (SKCKOLONPI GD, totally $180 \mu \mathrm{m}$ including adhesive), as shown in Fig. 4f. Rolled-annealed (RA) copper was chosen instead of electrodeposited (ED) due to the robustness against crack initiation and crack propagation ${ }^{55}$.

According to our latest design, each facet's circuits were rearranged to be interconnected by 4 or 6 parallel connecting lines over the creases (Fig. $10 \mathrm{~b})$. There was only one metal layer in the crease areas, making them treated as a single-layer FPCB (Fig. 10c). Furthermore, inspired by the concept kirigami, materials around the vertexes were entirely removed to have the strain redistributed on the MO-SCB and provide more DOFs during deformation ${ }^{60}$. Thickness reduction and structural weakening in the crease areas facilitated the stiffness modification, and the bending stiffness is evaluated in Supplementary Note $5^{61}$.

Figure $10 \mathrm{~d}$ shows the process of making MO-SCB. All the components were mounted on the top side of the board. Optionally, the components were sealed with glob-top compounds to avoid damage during the mold pressing. Next, we shaped the board into a Miura-ori structure through a two-stage folding process: (stage-1 folding) two sets of molds, each including a pair of top and bottom molds (Fig. 10e, detailed in Supplementary Note 13), forced the board to undergo an initial deformation; (stage-2 folding) heat shrink film (POF, polyolefin) helped to fold the board further. Due to the spring-back property of the $\mathrm{FPCB}^{62}$, the folding degrees $\varphi$ after stage- 1 and stage- 2 folding are about $6^{\circ}$, and $21^{\circ}$ (as the stable folding state, $\varphi_{\mathrm{m}}$ ), respectively Finally, the MO-SCB was packaged with an elastomer (Ecoflex $\left.{ }^{\mathrm{TM}} 00-30\right)$ colored by the pigment (SilcPig ${ }^{\text {TM }}$ PMS Black), as in Fig. $10 f$. 


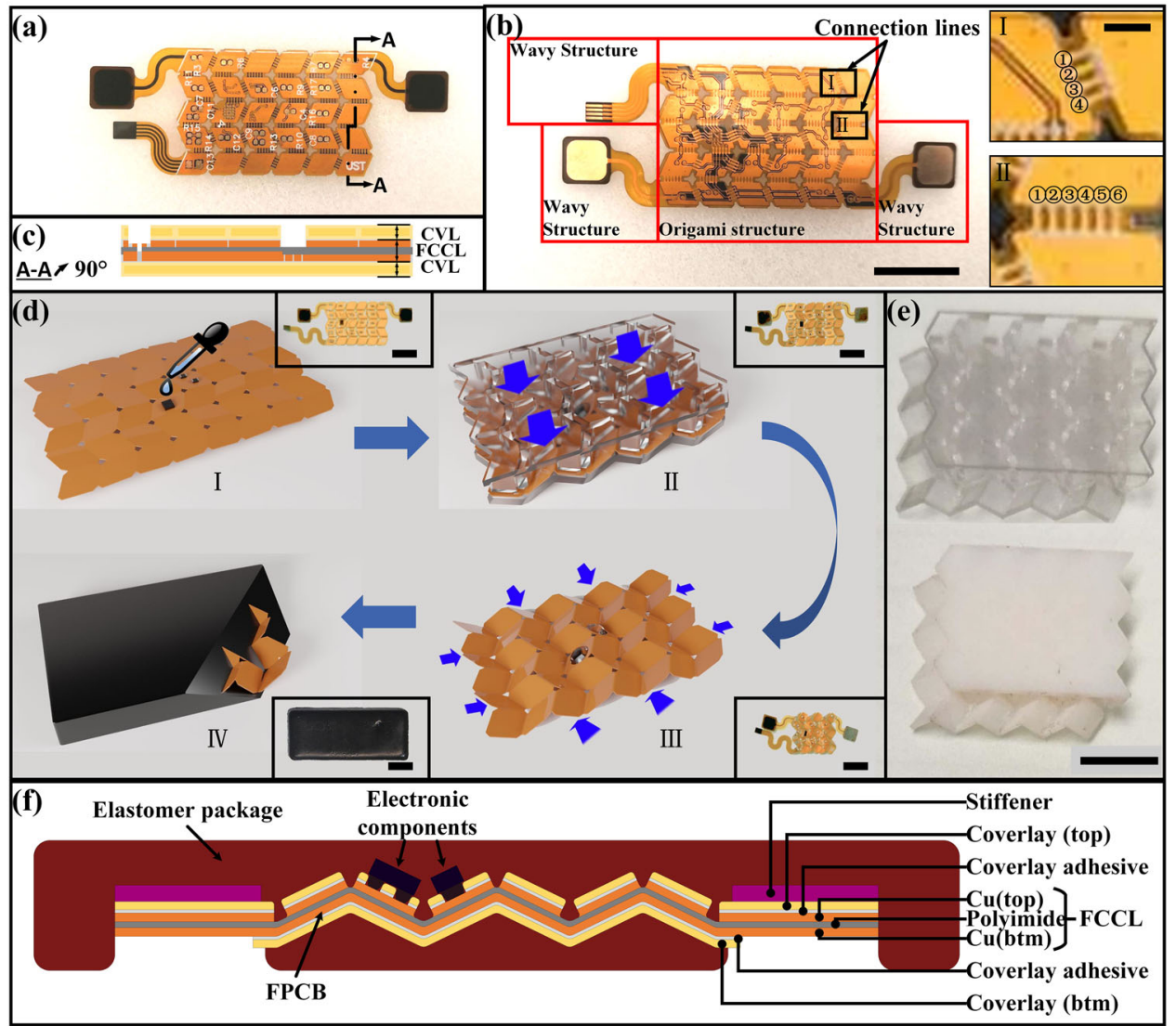

Fig. 10 Schematic diagram of the process of making a MO-SCB from an FPCB. a-c Without a folding process, i.e., in the fully unfolded state. View from a top, b bottom, and c cross-section along A-A (not to scale). Wavy lines serve as connections between the board and electrodes. The longer one is used for power (VCC/GND) and output (OUT/LOD) function. The other pair of shorter wires are used for ECG signal collection. The parallelogram facets are interconnected by four (inset I: for major creases) or six parallel (inset II: for minor creases) connecting wires over the creases. LOD lead-off detection. d Processes and the real MO-SCB corresponding to the processes. (I) (optional) sealing with glob-top; (II) stage-1 folding; (III) stage-2 folding; (IV) elastomer packaging. e Molds help in shaping the origami-structured parts. The folding degrees of the 1 st-level (transparent) and 2 nd-level (white) molds are $10^{\circ}$ and $24^{\circ}$. $\mathbf{f}$ Schematic of the cross-section of a packaged MO-SCB. (not to scale). Scale bars in $\mathbf{b}, \mathbf{e}$, and the insets of $\mathbf{d}$ represent $10 \mathrm{~mm}$, while that in the insets of $\mathbf{b}$ is $1 \mathrm{~mm}$.

\section{DATA AVAILABILITY}

The data that support the findings of this study are available from the authors on reasonable request. The authors declare that the data supporting this study's findings are available within the article and the corresponding supplementary information files.

\section{CODE AVAILABILITY}

This research does not have custom code or mathematical algorithm that is deemed central to the conclusions.

Received: 19 September 2020; Accepted: 21 January 2021; Published online: 15 February 2021

\section{REFERENCES}

1. Hong, Y. J., Jeong, H., Cho, K. W., Lu, N. \& Kim, D. H. Wearable and implantable devices for cardiovascular healthcare: from monitoring to therapy based on flexible and stretchable electronics. Adv. Funct. Mater. 29, 1808247 (2019).

2. Vieroth, R. et al. Stretchable circuit board technology and application. in 2009 International Symposium on Wearable Computers. 33-36 (IEEE, 2009).

3. Yamamoto, D. et al. A planar, multisensing wearable health monitoring device integrated with acceleration, temperature, and electrocardiogram sensors. Adv. Mater. Technol. 2, 1700057 (2017).

4. Yoon, J. et al. Soft modular electronic blocks (SMEBs): a strategy for tailored wearable health-monitoring systems. Adv. Sci. 6, 1801682 (2019).
5. Huang, Z. et al. Three-dimensional integrated stretchable electronics. Nat. Electron. 1, 473-480 (2018).

6. Byun, J. et al. A single droplet-printed double-side universal soft electronic platform for highly integrated stretchable hybrid electronics. Adv. Funct. Mater. 27, 1701912 (2017).

7. Gray, D. S., Tien, J. \& Chen, C. S. High-conductivity elastomeric electronics. Adv. Mater. 16, 393-397 (2004).

8. Jiang, Q. et al. Flexible and multi-form solid-state supercapacitors based on polyaniline/graphene oxide/CNT composite films and fibers. Diam. Relat. Mater. 92, 198-207 (2019).

9. Kim, B. S. et al. 2D reentrant auxetic structures of graphene/CNT networks for omnidirectionally stretchable supercapacitors. Nanoscale 9, 13272-13280 (2017).

10. Lee, D. W. \& Li, G. Stretchable circuit board and method of manufacturing the same. US patent 10057981B2 (2016).

11. Kim, H.-J., Son, C. \& Ziaie, B. A multiaxial stretchable interconnect using liquidalloy-filled elastomeric microchannels. Appl. Phys. Lett. 92, 011904 (2008).

12. Ghaffarzadeh, K. Stretchable electronics: near term commercial success stories. IDTechEx Res. https://www.idtechex.com/en/research-article/stretchableelectronics-near-term-commercial-success-stories/11170 (2017).

13. Li, T., Suo, Z., Lacour, S. P. \& Wagner, S. Compliant thin film patterns of stiff materials as platforms for stretchable electronics. J. Mater. Res. 20, 3274-3277 (2005).

14. Lee, W. et al. Two-dimensional materials in functional three-dimensional architectures with applications in photodetection and imaging. Nat. Commun. 9, 1417 (2018).

15. Zhang, Y., Huang, Y. \& Rogers, J. A. Mechanics of stretchable batteries and supercapacitors. Curr. Opin. Solid State Mater. Sci. 19, 190-199 (2015).

16. Hu, X., Dou, Y., Li, J. \& Liu, Z. Buckled structures: fabrication and applications in wearable electronics. Small 15, 1804805 (2019). 
17. Song, Z. et al. Origami lithium-ion batteries. Nat. Commun. 5, 3140 (2014).

18. Lynd, D. T. \& Harne, R. L. Strategies to predict radiated sound fields from foldable, Miura-ori-based transducers for acoustic beamfolding. J. Acous. Soc. Am. 141, 480-489 (2017).

19. Tang, R. et al. Origami-enabled deformable silicon solar cells. Appl. Phys. Lett. 104, 083501 (2014).

20. Gardner, J. P. et al. The James Webb space telescope. Space Sci. Rev. 123, 485-606 (2006).

21. Iwata, Y. \& Iwase, E. Stress-free stretchable electronic device using folding deformation. in 2017 IEEE 30th International Conference on Micro Electro Mechanical Systems (MEMS). 231-234 (IEEE, 2017).

22. Xiang, X., Lu, G., Ruan, D., You, Z. \& Zolghadr, M. Large deformation of an arcMiura structure under quasi-static load. Compos. Struct. 182, 209-222 (2017).

23. Dieleman, P., Vasmel, N., Waitukaitis, S. \& van Hecke, M. Jigsaw puzzle design of pluripotent origami. Nat. Phys. 16, 63-68 (2020).

24. Evans, T. A., Lang, R. J., Magleby, S. P. \& Howell, L. L. Rigidly foldable origami twists. Origami 6, 119-130 (2015).

25. Evans, T. A., Lang, R. J., Magleby, S. P. \& Howell, L. L. Rigidly foldable origami gadgets and tessellations. R. Soc. Open Sci. 2, 150067 (2015).

26. Sareh, P. The least symmetric crystallographic derivative of the developable double corrugation surface: Computational design using underlying conic and cubic curves. Mater. Des. 183, 108128 (2019).

27. Callens, S. J. P. \& Zadpoor, A. A. From flat sheets to curved geometries: origami and kirigami approaches. Mater. Today 21, 241-264 (2018).

28. Kasahara, K. \& Takahama, T. Origami for the Connoisseur 7th edn. (Japan Pubns, 1998).

29. Hull, T. in Origami 3: Third International Meeting of Origami Science, Mathematics, and Education. 1st edn. (ed. Thomas Hull) 29-38 (A K Peters/CRC Press, 2002).

30. McAdams, D. A. \& Li, W. A novel method to design and optimize flat-foldable origami structures through a genetic algorithm. J. Comput. Inf. Sci. Eng. 14, 031008 (2014).

31. Tachi, T. Generalization of rigid-foldable quadrilateral-mesh origami. J. Int. Assoc. Shell Spat. Struct. 50, 173-179 (2009).

32. Fonseca, L. M., Rodrigues, G. V., Savi, M. A. \& Paiva, A. Nonlinear dynamics of an origami wheel with shape memory alloy actuators. Chaos Solitons Fractals 122, 245-261 (2019).

33. Brunck, V., Lechenault, F., Reid, A. \& Adda-Bedia, M. Elastic theory of origamibased metamaterials. Phys. Rev. E 93, 033005 (2016).

34. Kawasaki, T. \& Yoshida, M. Crystallographic flat origamis. Mem. Fac. Eng. Kyushu Univ. Ser. A 42, 153-157 (1988).

35. Zirbel, S. A. et al. Accommodating thickness in origami-based deployable arrays. J. Mech. Des. 135, 111005 (2013).

36. Lang, R. J., Tolman, K. A., Crampton, E. B., Magleby, S. P. \& Howell, L. L. A review of thickness-accommodation techniques in origami-inspired engineering. Appl Mech. Rev. 70, 010805 (2018).

37. Huang, Z., Hong, W. \& Suo, Z. Nonlinear analyses of wrinkles in a film bonded to a compliant substrate. J. Mech. Phys. Solids 53, 2101-2118 (2005).

38. Huang, Z., Hong, W. \& Suo, Z. Evolution of wrinkles in hard films on soft substrates. Phys. Rev. E 70, 030601 (2004).

39. Liu, K. \& Paulino, G. Nonlinear mechanics of non-rigid origami: an efficient computational approach. Proc. Math. Phys. Eng. Sci. 473, 20170348 (2017).

40. Liu, S., Lu, G., Chen, Y. \& Leong, Y. W. Deformation of the Miura-ori patterned sheet. Int. J. Mech. Sci. 99, 130-142 (2015).

41. Terada, Y., Sakamoto, H. \& Okuma, M. Characteristics of deployable planar origami structures with partial constraints. in 2018 AlAA Spacecraft Structures Conference. 2205 (American Institute of Aeronautics and Astronautics Inc, 2018).

42. Fang, H., Wang, K. W. \& Li, S. Asymmetric energy barrier and mechanical diode effect from folding multi-stable stacked-origami. Extrem. Mech. Lett. 17, 7-15 (2017).

43. Tolman, K. A., Lang, R. J., Magleby, S. P. \& Howell, L. L. Split-vertex technique for thickness-accommodation in origami-based mechanisms. in ASME 2017 International Design Engineering Technical Conferences and Computers and Information in Engineering Conference. Vol. 58189, V05BT08A054 (American Society of Mechanical Engineers, 2017).

44. Mao, L., Meng, Q., Ahmad, A. \& Wei, Z. Mechanical analyses and structural design requirements for flexible energy storage devices. Adv. Energy Mater. 7, 1700535 (2017).

45. Zhao, D., Li, Y., Wang, M., Duan, C. \& You, Z. Fabrication of polymer origamibased V-type folded core. in ASME 2015 International Design Engineering Technical Conferences and Computers and Information in Engineering Conference. Vol. 57137, V05BT08A038 (American Society of Mechanical Engineers, 2015).

46. Victor, G. L. Process and devices for chevroning pliable sheet material. US patent 3397261 (1968)
47. Zirbel, S. A., Wilson, M. E., Magleby, S. P. \& Howell, L. L. An origami-inspired selfdeployable array. in ASME 2013 Conference on Smart Materials, Adaptive Structures and Intelligent Systems. Vol. 56031, V001T001A026 (American Society of Mechanical Engineers, 2013).

48. Lin, C.-H. et al. Highly deformable origami paper photodetector arrays. ACS Nano 11, 10230-10235 (2017)

49. Zuliani, F., Liu, C., Paik, J. \& Felton, S. M. Minimally actuated transformation of origami machines. IEEE Robot. Autom. Lett. 3, 1426-1433 (2018).

50. Cai, J. et al. Deployment simulation of Miura-origami membrane structures. in ASME 2016 International Design Engineering Technical Conferences and Computers and Information in Engineering Conference. Vol. 50169, V05BT07A002 (American Society of Mechanical Engineers, 2016).

51. Wilson, L., Pellegrino, S. \& Danner, R. Origami sunshield concepts for space telescopes. in 54th AIAA/ASME/ASCE/AHS/ASC Structures, Structural Dynamics, and Materials Conference. 1594 (2013).

52. Cowan, B. \& von Lockette, P. R. Fabrication, characterization, and heuristic trade space exploration of magnetically actuated Miura-ori origami structures. Smart Mater. Struct. 26, 045015 (2017).

53. Schenk, M. \& Guest, S. D. Geometry of Miura-folded metamaterials. Proc. Natl Acad. Sci. USA 110, 3276-3281 (2013).

54. Schenk, M. \& Guest, S. D. in Origami 5: Fifth International Meeting of Origami Science, Mathematics, and Education. 1st edn. (eds. Patsy Wang-Iverson, Robert J. Lang, \& Mark Yim) 291-304 (A K Peters/CRC Press, 2016).

55. Coonrod, J. Bending, forming and flexing printed circuits. Rogers Corporation Technical Data Sheet http://magazines007.com/pdf/Bending-FormingFlexingPCBs.pdf (2018).

56. Eidini, M. \& Paulino, G. H. Unraveling metamaterial properties in zigzag-base folded sheets. Sci. Adv. 1, e1500224 (2015).

57. Silverberg, J. L. et al. Origami structures with a critical transition to bistability arising from hidden degrees of freedom. Nat. Mater. 14, 389 (2015).

58. Sareh, P. \& Chen, Y. Intrinsic non-flat-foldability of two-tile DDC surfaces composed of glide-reflected irregular quadrilaterals. Int. J. Mech. Sci. 185, 105881 (2020).

59. Yoo, J., Yan, L., Lee, S., Kim, H. \& Yoo, H.-J. A wearable ECG acquisition system with compact planar-fashionable circuit board-based shirt. IEEE Trans. Inf. Technol. Biomed. 13, 897-902 (2009).

60. Saito, K., Tsukahara, A. \& Okabe, Y. Designing of self-deploying origami structures using geometrically misaligned crease patterns. Proc. Math. Phys. Eng. Sci. 472, 20150235 (2016).

61. Nettles, A. T. Basic mechanics of laminated composite plates. NASAR https://ntrs. nasa.gov/citations/19950009349 (1994).

62. Chang, J.-Y. Mechanics of flexible interconnect in lateral tape open drives. Microsyst. Technol. 18, 1513-1516 (2012).

\section{ACKNOWLEDGEMENTS}

We wish to thank Yik Kin Cheung for rendering 3D pictures and Tracy Pei for manuscript revision and discussion. This work was supported by grants from the Innovation and Technology Commission (project ITS/051/18) of HKSAR, National Natural Science Foundation of China (NSFC, project: 11932009), Foshan HKUST Projects (Grant No.: FSUST19-FYTRI05), and the startup fund from the Hong Kong University of Science and Technology. We would like to acknowledge the Nanosystem Fabrication Facility (NFF) of the Hong Kong University of Science and Technology and appreciate Lillian Yuan and Mark Ellwood for proofreading and English editing.

\section{AUTHOR CONTRIBUTIONS}

Y.L. and Y.D. conceived the idea, developed the methods, and designed the experiments under the supervision of H.Y. W.L. performed the simulation under the supervision of W.H. Y.L. performed the experiments, analyzed the data, and wrote the paper under the supervision of H.Y. and W.H. Y.L. and W.L. contributed equally to this work.

\section{COMPETING INTERESTS}

The authors declare no competing interests.

\section{ADDITIONAL INFORMATION}

Supplementary information The online version contains supplementary material available at https://doi.org/10.1038/s41528-021-00099-8.

Correspondence and requests for materials should be addressed to W.H. or H.Y 
Reprints and permission information is available at http://www.nature.com/ reprints

Publisher's note Springer Nature remains neutral with regard to jurisdictional claims in published maps and institutional affiliations.

(c) Open Access This article is licensed under a Creative Commons Attribution 4.0 International License, which permits use, sharing, adaptation, distribution and reproduction in any medium or format, as long as you give appropriate credit to the original author(s) and the source, provide a link to the Creative Commons license, and indicate if changes were made. The images or other third party material in this article are included in the article's Creative Commons license, unless indicated otherwise in a credit line to the material. If material is not included in the article's Creative Commons license and your intended use is not permitted by statutory regulation or exceeds the permitted use, you will need to obtain permission directly from the copyright holder. To view a copy of this license, visit http://creativecommons. org/licenses/by/4.0/.

(c) The Author(s) 2021 